\title{
Effect of 8.4\% Soda-Bicarbonate Steam Inhalation on the Course of Disease in Mild to Moderate Cases of Covid-19
}

\section{Kshitij Mody*}

Department of Orthopaedics, Welcare Hospital, India

*Corresponding Author: Kshitij Mody, Department of Orthopaedics, Welcare Hospital, India.
Received: February 26, 2021

Published: March 19, 2021

(C) All rights are reserved by Kshitij Mody.

\begin{abstract}
Background: A prospective, randomized open label parallel group trial was carried out to evaluate the effect of $8.4 \%$ soda-bicarbonate steam inhalation on the course of covid-19 infection in mild to moderate confirmed cases of covid-19.

Methods: 30 patients were enrolled and compared with 30 patients in a control group, giving a total sample size of 60 patients. Randomisation was achieved by sealed envelope technique. The 30 patients in the SB group received SB inhalation in addition to all other therapeutic measures as part of covid-19 treatment protocol. The control group was treated according to same treatment protocol, but without SB inhalation therapy. Clinical symptoms and lab markers were recorded on Day 1 and Day 5 of patients' stay at hospital. Results: Patients receiving 8.4\% SB inhalation as part of their treatment showed faster improvement in symptomatology and quicker normalization of inflammatory lab markers.

Conclusion: The results of this study show a highly significant improvement in the clinical picture of covid-19 affected patients treated with inhalations of steam impregnated with $8.4 \%$ sodium bicarbonate.
\end{abstract}

Keywords: Covid-19; Sodium Bicarbonate; Steam Inhalation

\section{Abbreviations}

ASL: Airway Surface Liquid; SB: Sodium Bicarbonate; RT-PCR: Reverse Transcription Polymerase Chain Reaction

\section{Introduction}

The human population of the world is currently facing a pandemic of respiratory infection. We know that such diseases can be caused by various microbes, including bacteria and fungi as well as viruses [1]. These organisms require certain environmental conditions to thrive and cause damage via the infection. A key factor amongst these conditions is the local $\mathrm{pH}$. The $\mathrm{pH}$ of the environment where these microbes harbour can significantly influence biological activity including enzyme actions, reaction rates, protein and nucleic acid stability $[2,3]$.
Associated with this infective activity are the properties of the airway surface liquid (ASL), which contains a complex mixture of antimicrobial factors that kill inhaled or aspirated organisms and act as a first line of defence. The composition of ASL is critical for antimicrobial effectiveness. Changes in the ASL occur with inflammation or infection, resulting in local acidosis [4,7].

In respiratory tract infections caused by bacteria, viruses, fungi, and mycobacteria, there is usually a local acidic medium in the lung secretions. Thus, the infection results in lowering of the local $\mathrm{pH}$ within the respiratory tract.

The present pandemic of Covid-19 is caused by a novel member of the Corona group of viruses, namely SARS-CoV-2;2019-nCoV. 
There is evidence in literature that viruses belonging to the Corona group need a low $\mathrm{pH}$ environment to allow the first connectivity of the virus to the cell wall [5-7]. It therefore follows that if the infection lowers the $\mathrm{pH}$, and in turn supports viral proliferation, any elevation of the $\mathrm{pH}$ of naso-oropharyngeal environment to an alkaline medium may disrupt the virus and its activity.

Sodium Bicarbonate (SB) inhalation (4.2\% and 8.4\%) has been used as a treatment measure in pathological respiratory conditions such as cystic fibrosis and chlorine gas inhalations. Its safety, tolerability and efficacy in improvement in those clinical conditions have been well established $[8,9]$.

We set out to evaluate the effect of SB 8.4\% inhaled with steam resulting in the increased pH of the ASL on the course of Covid-19 infection.

\section{Aim of the Study}

The aim was to find a method of aborting and/or mitigating the devastating effects of Covid-19 infection by means of an inexpensive, accessible and safe agent. The effect was monitored (i) clinically and (ii) by measuring the surrogate markers of the two processes (intense inflammation and coagulation) which have been identified as the method by which high morbidity and mortality results in these patients. These markers are ESR, CRP, Serum Ferritin, Serum Procalcitonin, Serum LDH, IL6 and D Dimer.

\section{Materials and Methods}

We carried out a prospective randomized trial, using an open label randomized parallel group method. The subjects were adults aged 18 or more, with clinical covid-19 disease confirmed by a RTPCR test. All patients were admitted in hospital wards receiving treatment according to standard covid-19 treatment protocol. We excluded patients on ventilatory support.

The study was subject to local ethical committee approval and patients were given a volunteer information document and signed an informed consent prior to enrolment.

After approval of the local ethical committee and registration of the study on Clinical Trials Registry of India with registration number (CTRI/2020/07/026535), all patients signed their written consents after detailed explanation of the study protocol.

\section{Study group}

30 patients were enrolled (SB group) and compared with 30 patients in a control group (Non-SB group), giving a total study size of 60 patients. Randomisation was achieved by sealed envelope technique. The 30 patients in the SB group received $8.4 \%$ sodium bicarbonate steam inhalation in addition to all other therapeutic measures as part of covid-19 treatment protocol. The control group was treated according to same treatment protocol, but without $8.4 \%$ sodium bicarbonate steam inhalation therapy.

Administration of sodium bicarbonate and measurement of pH

For each administration, $50 \mathrm{ml}$ of $8.4 \%$ Sodium Bicarbonate vapour was delivered through a naso-oral delivery apparatus attached to an electrically operated steam generating apparatus (See figure 1). The administration was for 5 minutes per session. This was done at a frequency of twice daily 8 hours apart, for 5 days.

After each administration of $8.4 \%$ SB steam, the $\mathrm{pH}$ of the patient's saliva was measured using a $\mathrm{pH}$ indicator strip.

\section{Outcome measures}

Outcome was assessed by the following:

1. Clinical parameters. The clinical progression of patient's condition was monitored and recorded using these criteria: Fever, Cough, Sore throat, Runny nose, Wheezing, Chest pain, Muscle aches, Joint pain, Fatigue/Malaise, Shortness of breath, inability to walk, Headache, Lover chest wall indrawing, Altered consciousness/confusion, Seizures, Abdominal pain, Vomiting/Nausea, Diarrhoea, Conjunctivitis, Skin rash, Skin ulcers, Lymphadenopathy, Bleeding (Haemorrhage), Loss of taste and Loss of smell.

2. Laboratory markers:
a. ESR
b. CRP
c. Serum Ferritin
d. Serum Procalcitonin
e. Serum LDH
f. Serum IL6
g. D-dimer. 
ESR, CRP, S. ferritin, S. procalcitonin, S. LDH and S. IL6 are markers of infection and inflammation while D-dimer is a marker of coagulopathy.

The higher the values of these lab markers, the more intense the underlying pathological process.

\section{Statistical analysis}

The statistical analysis of data was done using R software version 4.0.3. The data was categorically presented with numbers and percentages. Chi-square (or Fisher's Exact Test when needed) were used to compare the results between the SB group and nonSB group. Continuous data was presented descriptively with mean, median and standard deviation (SD). Comparison between the SB and the non-SB group for change from baseline in different parameters was done using two-sample t-test. All the statistical tests performed were two sided with a level of significance of 5\%. Within group (SB and non-SB group) comparisons for the change in different parameters was done using the paired t-test. Data was also presented graphically using spaghetti plots for individual subjects for all the parameters.

\section{Results}

Each laboratory parameter was summarized descriptively by Day (Day 1 and Day 5) and also by group (SB and Non-SB group). This descriptive summary included mean, median, standard deviation, minimum and maximum.

Change of lab values from baseline data, from Day 1 to Day 5, was also summarized descriptively.

On summarising the change from baseline data, we found that the change from Day 1 to Day 5 is numerically higher in the SB group than that for the non-SB group for the following parameters - ESR, CRP, LDH, IL6, D-Dimer and Ferritin.

For most patients in the SB group, this change was in the form of reduction in the lab values of markers (ESR, CRP, LDH, IL6 and Ferritin) from Day 1 to Day 5 thus indicating an improvement in the underlying process of inflammation/infection. Spaghetti plots for individual subjects for all the parameters confirmed the trend of improvement in the SB group as compared to the non-SB group.
Spaghetti plots for day 1 to day 5 progression of laboratory parameters

Similarly, the change of D-dimer values from day 1 to day 5 in most patients in the SB group was in the form of reduction in lab values thus indicating an improvement in the underlying pathological process of coagulopathy.
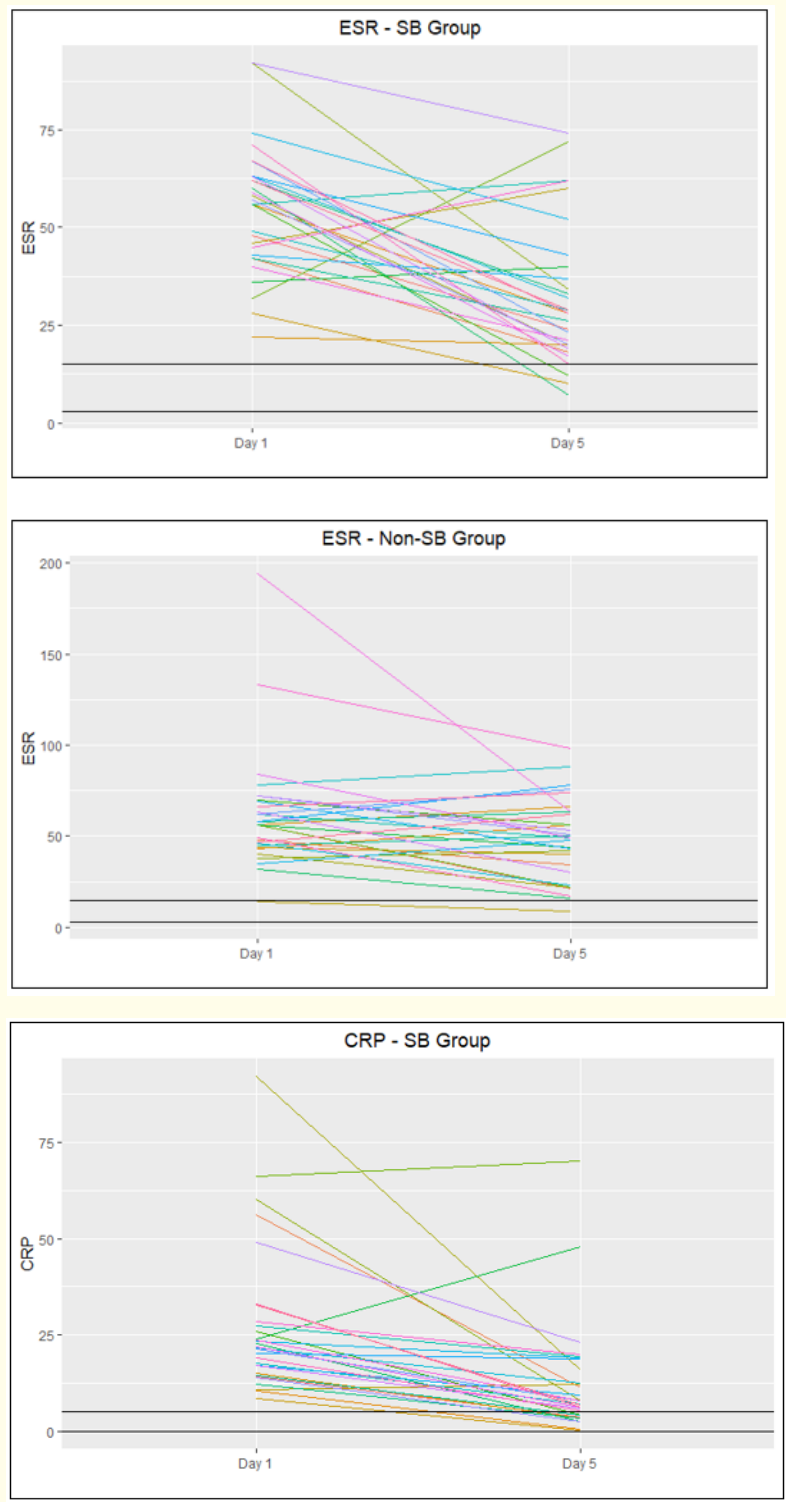

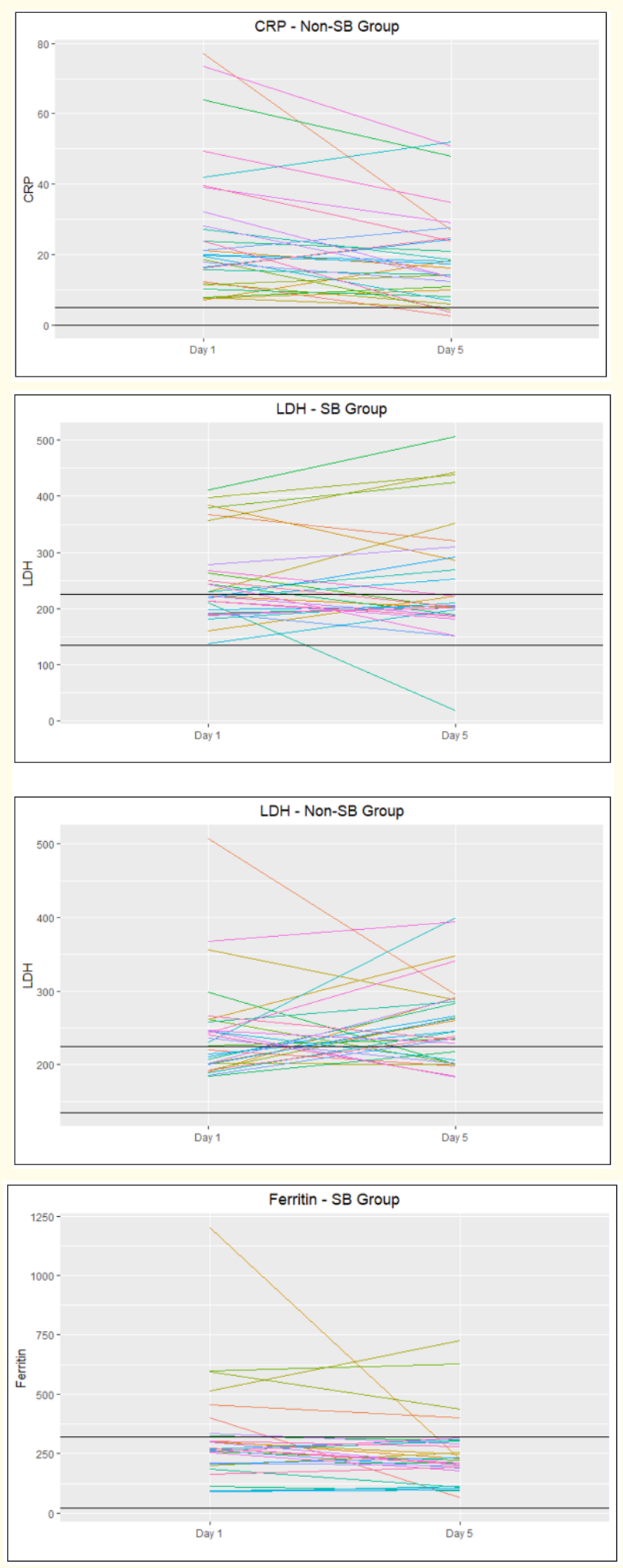
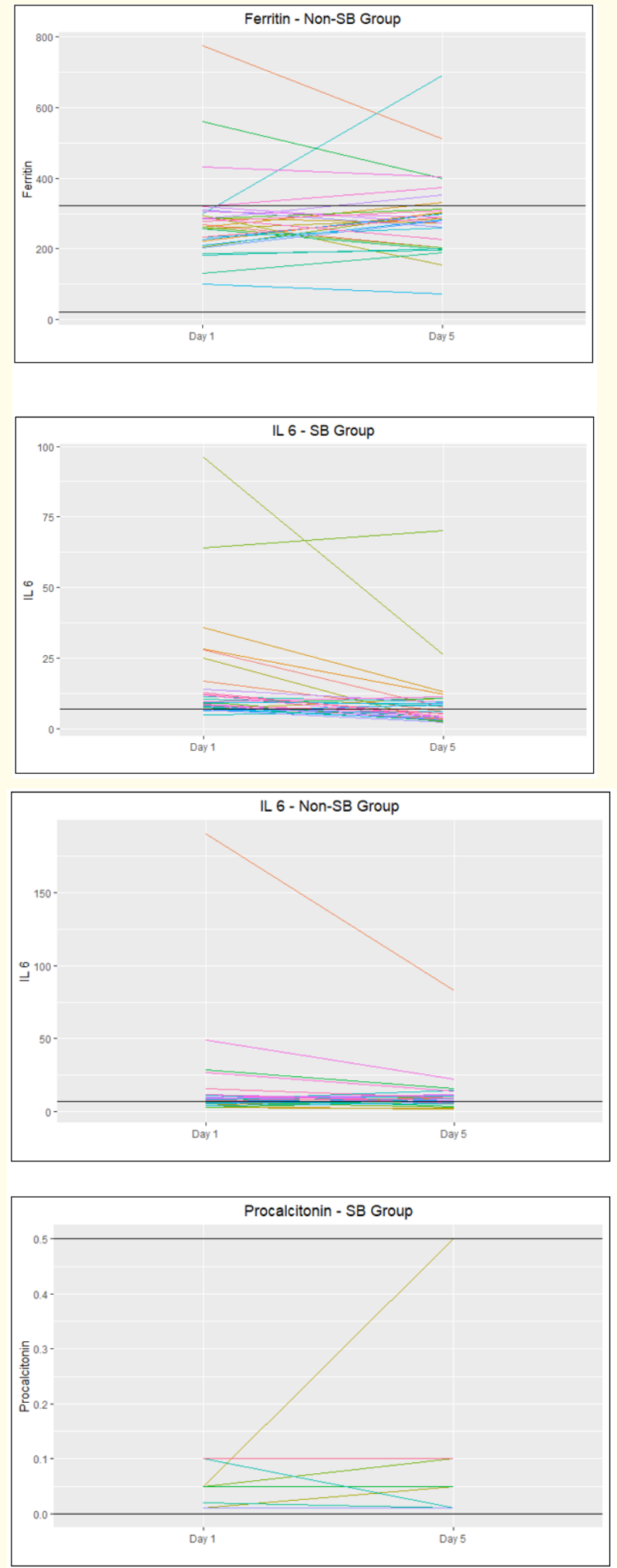


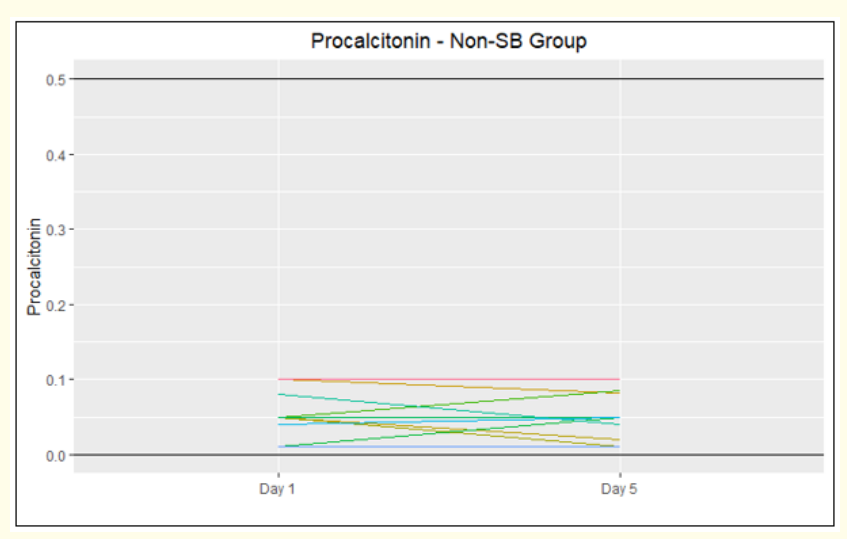

Graph 1
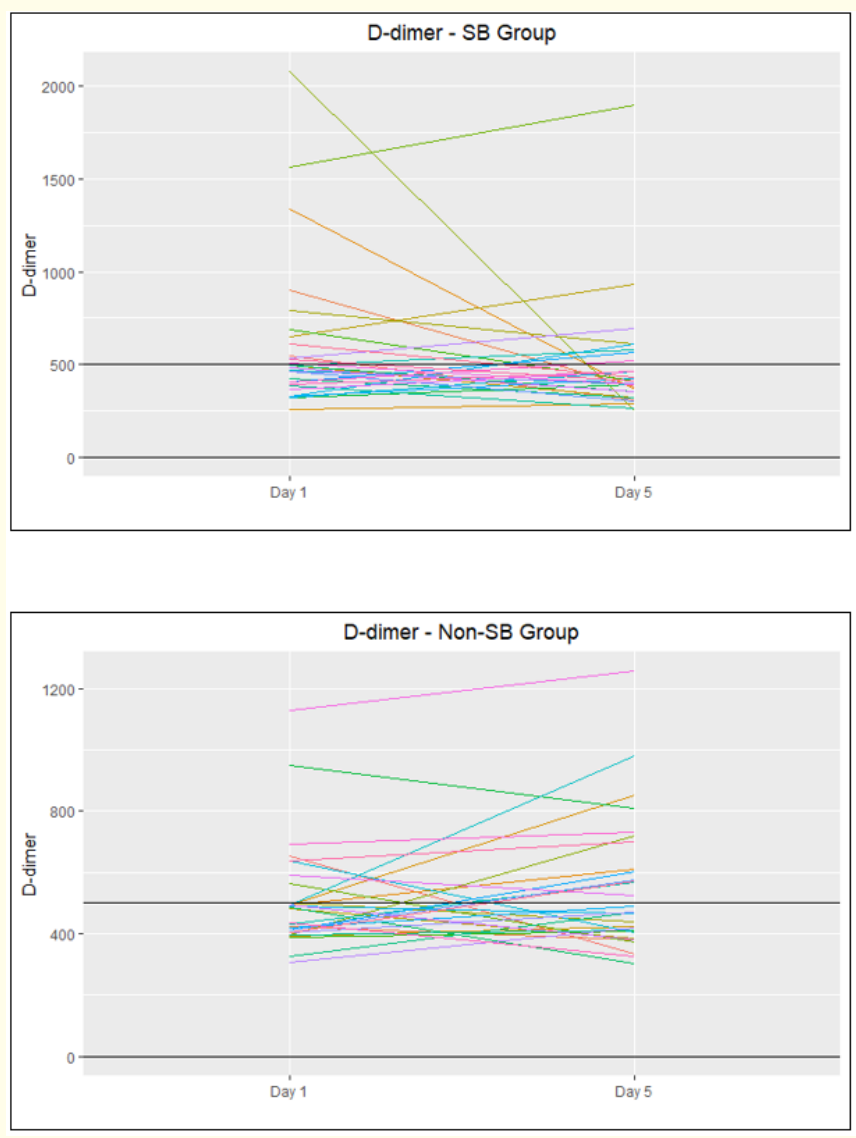

Graph 2
For CRP, the change from baseline to Day 5 was statistically significantly better in the SB group than the non-SB group ( $p$-value 0.043).

The overall small sample size of the study could be one reason that the difference between the two treatments in terms of change from baseline could not show statistical significance for ESR, LDH, IL6, Ferritin and D-Dimer. However, as mentioned above, for all these parameters the results are numerically better in the SB group.

The p-values for each parameter for within treatment group comparison (i.e. the change in parameter values from Day 1 to Day 5 for SB and non-SB groups separately) have been shown in the table below (Table 1). P-values less than 0.05 have been considered to be statistically significant.

Proportion of subjects showing improvement was statistically significantly higher in the SB group as compared to the non-SB group as shown in the table below (Table 2). Proportion of improvement was found to be 73.33 and 26.67 in the SB and Non-SB groups, respectively (p-value 0.0003).

\section{Discussion}

Infection by the Covid-19 virus poses significant dangers to a patient's life, and any therapeutic measure that can influence that favourably should be a welcome addition to the therapeutic armamentarium. Whilst vaccines remain in various stages of development, patients continue to present and require treatment beyond the supportive.

Like other coronaviruses (SARS-CoV), entry of SARS-CoV-2 into a host cell seems to be $\mathrm{pH}$ dependent, because once a virus fuses with a human cell via S- glycoprotein then its entry inside the cell utilizes a pH-dependent endocytotic pathway [10]. When these endo-lysosome vesicles move towards the nucleus, their $\mathrm{pH}$ drops (more acidic), which catalyzes fusion of viral and cell membranes [11]. The studies have mentioned that there is more reduction in viral entry if alkaline conditions are retained in the host cells i.e. $\mathrm{pH}$ $>7$, while as under acidified conditions $(\mathrm{pH}<7)$ there is more viral load inside the host cells $[10,12,13]$. Therefore, it is evident that novel therapeutic strategies could be designed to lower the $\mathrm{pH}$ (alkaline) of endo-lysosomes through infusion of $\mathrm{pH}$ lowering agents 


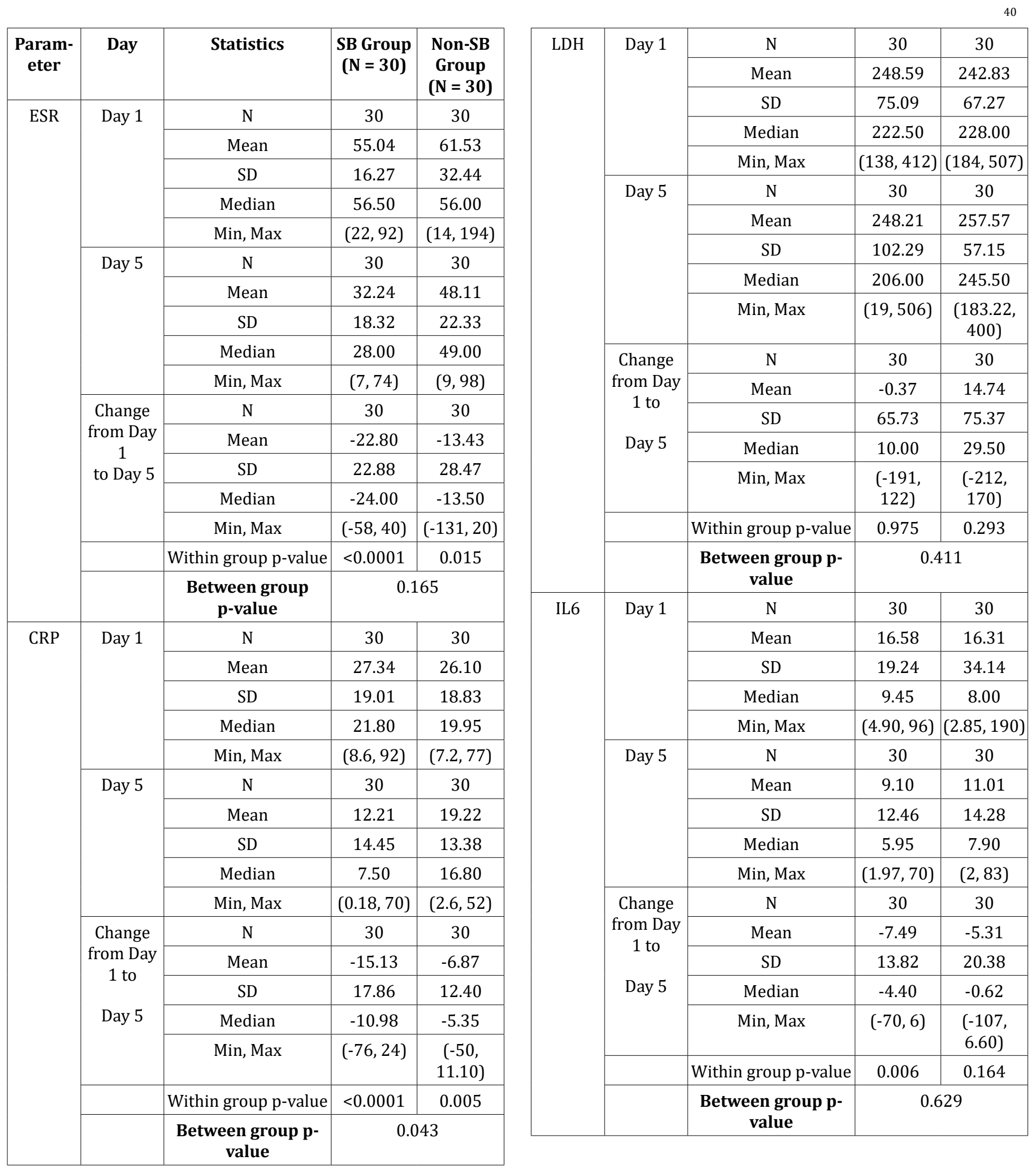




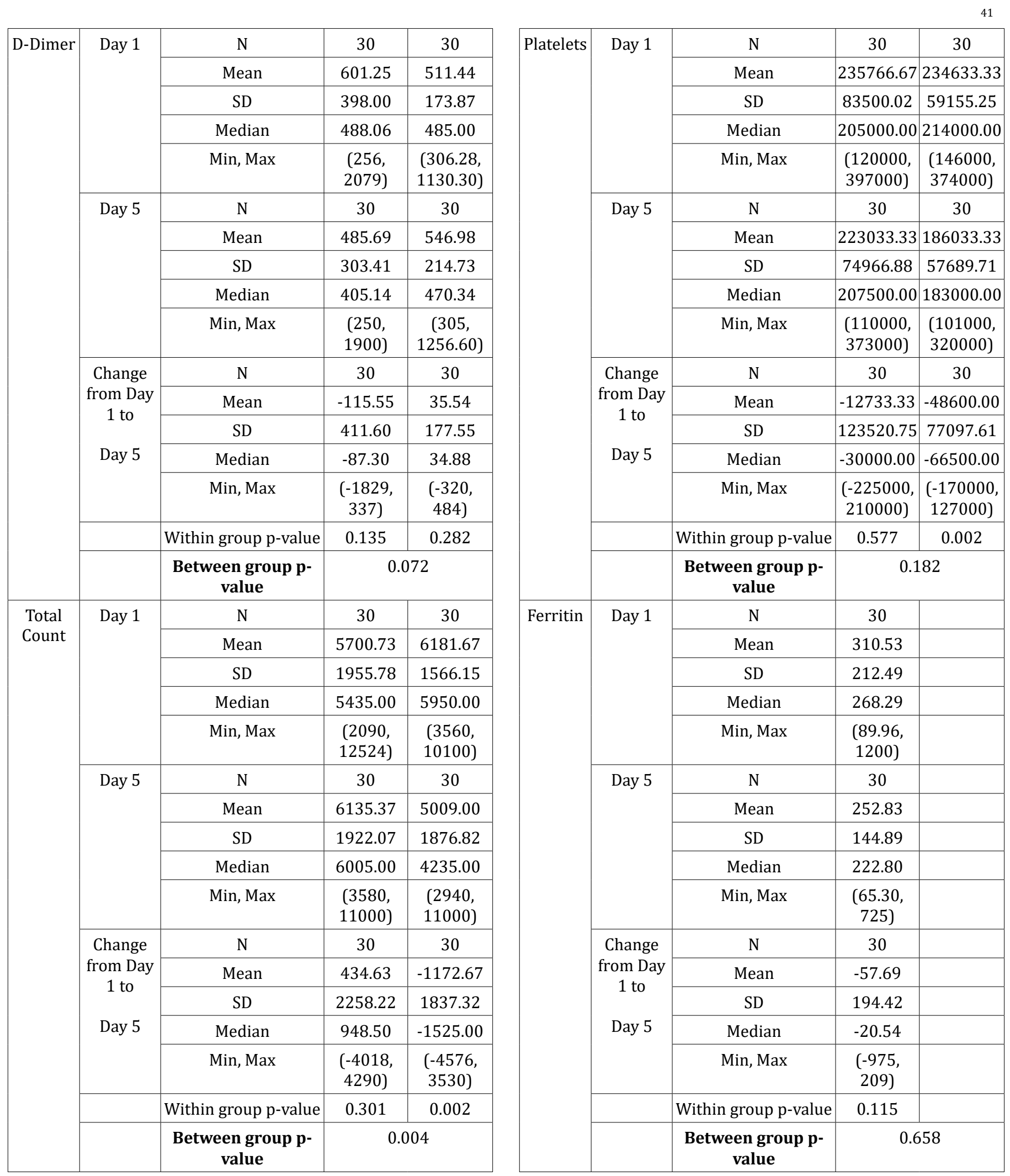




\begin{tabular}{|c|c|c|c|c|}
\hline \multirow{17}{*}{$\begin{array}{l}\text { Procalci- } \\
\text { tonin }\end{array}$} & \multirow[t]{5}{*}{ Day 1} & $\mathrm{~N}$ & 30 & \\
\hline & & Mean & 0.06 & \\
\hline & & SD & 0.04 & \\
\hline & & Median & 0.05 & \\
\hline & & Min, Max & $\begin{array}{l}(0.01 \\
0.10)\end{array}$ & \\
\hline & \multirow[t]{5}{*}{ Day 5} & $\mathrm{~N}$ & 30 & \\
\hline & & Mean & 0.07 & \\
\hline & & SD & 0.09 & \\
\hline & & Median & 0.05 & \\
\hline & & Min, Max & $\begin{array}{l}(0.01, \\
0.50)\end{array}$ & \\
\hline & \multirow{5}{*}{$\begin{array}{l}\text { Change } \\
\text { from Day } \\
1 \text { to }\end{array}$} & $\mathrm{N}$ & 30 & \\
\hline & & Mean & 0.01 & \\
\hline & & SD & 0.08 & \\
\hline & & Median & 0.00 & \\
\hline & & Min, Max & $\begin{array}{c}(-0.09 \\
0.45)\end{array}$ & \\
\hline & & Within group p-value & 0.351 & \\
\hline & & $\begin{array}{l}\text { Between group p- } \\
\text { value }\end{array}$ & \multicolumn{2}{|c|}{0.121} \\
\hline
\end{tabular}

Table 1

\begin{tabular}{|c|c|c|c|c|}
\hline \multicolumn{2}{|c|}{ Parameter } & $\begin{array}{c}\text { SB Group } \\
(\mathbf{N}=30)\end{array}$ & $\begin{array}{c}\text { Non-SB Group } \\
\text { (N = 30)n (\%) }\end{array}$ & \multirow{2}{*}{ P-value } \\
\hline Improvement & Yes & $22(73.33)$ & $8(26.67)$ & \multirow{2}{*}{0.0003} \\
\cline { 2 - 4 } & No & $8(26.67)$ & $22(73.33)$ & \\
\hline
\end{tabular}

Table 2

known as lysosomotropic agents. They are defined as weaker bases that have potential to penetrate lysosomes in their protonated form and thus increase their intracellular $\mathrm{pH}$ [14]. The use of safer lysosomotropic agents could pave a way to act as one of the effective counter strategies to thwart infection caused by SARS-CoV-2.

This study has attempted to exploit the vulnerability of the virus to an alkaline $\mathrm{pH}$, which may offer a quick, cheap and readily available attempt to influence the course of the disease. Using simply inhalation of sodium bicarbonate (baking soda) solution in water, we demonstrated an elevation of the naso-oropharyngeal $\mathrm{pH}$, and a similar effect can be expected further down the respiratory tree. We have attempted to demonstrate the effect of doing so on the course of disease in patients who are hospitalised with positive diagnoses of covid-19 infection.

The results of this study showed a highly significant improvement in the clinical symptomatic picture of covid-19 affected patients when they were treated with inhalations of steam impregnated with $8.4 \%$ sodium bicarbonate. There was also a highly significant reduction in the CRP values of patients treated with steam inhalations impregnated with $8.4 \%$ sodium bicarbonate. Whilst there was a trend showing improvements in numerical values of other blood markers (ESR, LDH, IL6, D-dimer and Ferritin), they did not reach statistical significance perhaps due to the small sample size of the patients. The overall small sample size of the study could be one reason that the difference between the two treatments in terms of change from baseline could not show statistically significant results for ESR, LDH, IL6, D-Dimer and Ferritin. However, as mentioned above, for all these parameters the results are numerically better in the SB group.

The results of this study appear to validate the basic science findings that the Corona-Sars-2-enveloped virus-related activity can be mitigated by creating an alkaline $\mathrm{pH}$ environment in the nasopharyngeal and oropharyngeal spaces. There are recent reports [15] which indicate that combating this virus at the nasopharynx and oropharynx level in the early stages of the infection, or perhaps even before the stage of infection is reached can either prevent the infection, or failing which can reduce the severity of the disease.

Further studies with a larger patient population can establish further proof for the application of sodium bicarbonate as a useful agent to combat the Covid-19 infection.

\section{Conclusion}

There is a significant reduction in severity of symptoms of mild to moderate cases of covid-19 treated with $8.4 \%$ sodium bicarbonate steam inhalation as an adjuvant to standard covid treatment protocol. 
Proportion of subjects showing clinical symptomatic improvement was statistically significantly higher in the SB group as compared to the non-SB group. Proportion of improvement was found to be 73.33 and 26.67 in the SB and non-SB groups respectively (p-value 0.0003).

Further studies with a larger patient population can establish further proof for the application of sodium bicarbonate as a useful agent to combat the covid-19 infection.

\section{Acknowledgements}

I would like to acknowledge Myself Health Check Ltd (Unit 1 Hayes Estate, Godstone Road, Caterham, Surrey, United Kingdom CR36SF) for sponsoring this clinical study and providing an opportunity to add scientific value to the fight against Covid-19.

\section{Bibliography}

1. El Badrawy MK., et al. "Effect of sodium bicarbonate $8.4 \%$ on respiratory tract pathogens". The Journal of Chest and Lung Research 1.1 (2018): 3-7.

2. Alaiwa AMH., et al. "pH modulates the activity and synergism of the airway surface liquid antimicrobials defensin-3 and LL-37". Proceedings of the National Academy of Sciences of the United States of America 111.52 (2014): 18703-18708.

3. Slonczewski JL., et al. "Cytoplasmic pH measurement and homeostasis in bacteria and archaea". Advances in Microbial Physiology 55 (2009): 1-79.

4. Pezzulo AA., et al. "Reduced airway surface $\mathrm{pH}$ impairs bacterial killing in the porcine cystic fibrosis lung". Nature 487.7405 (2012): 109-113.

5. Yang ZY., et al. "pH-Dependent Entry of Severe Acute Respiratory Syndrome Coronavirus Is Mediated by the Spike Glycoprotein and Enhanced by Dendritic Cell Transfer through DCSIGN". Journal of Virology 78 (2004): 5642-5650.

6. Chu V McElroy., et al. "The Avian Coronavirus Infectious Bronchitis undergoes direct low PH dependent Fusion activation during entry into the host cells". Journal of Virology 80 (2006): 3180-3188.
7. Mudasir A Mir., et al. "A review on probable Lysosomotropic properties of Sodium bicarbonate to restrain viral entry of Coronavirus 2 (SARS-CoV-2) (2020).

8. Gomez CCS., et al. "Safety, tolerability and effects of sodium bicarbonate inhalation in cystic fi brosis". Clinical Drug Investigation 40 (2019): 105-117.

9. Aslan S., et al. "The effect of nebulized sodium bicarbonate treatment on RADS patients due to chlorine gas inhalation". Inhalation Toxicology 18 (2006): 895-900.

10. Yang ZY., et al. "pH-dependent entry of severe acute respiratory syndrome coronavirus is mediated by the spike glycoprotein and enhanced by dendritic cell transfer through DC-SIGN". Journal of Virology 78.11 (2004): 5642-5650.

11. Vincent R., et al. "Hydroxychloroquine reduces viral load in COVID-19 patients (2020).

12. Prajapat M., et al. "Drug targets for corona virus: A systematic review". Indian Journal of Pharmacology 52.1 (2020): 56.

13. Wang H., et al. "SARS coronavirus entry into host cells through a novel clathrin-and caveolae-independent endocytic pathway". Cell Research 18.2 (2008): 290-301.

14. Ashfaq UA., et al. "Lysosomotropic agents as HCV entry inhibitors". Virology Journal 8.1 (2011): 163.

15. Herrera D., et al. "Is the oral cavity relevant in SARS-CoV-2 pandemic?” Clinical Oral Investigations 24.8 (2020): 2925-2930.

\section{Assets from publication with us}

- Prompt Acknowledgement after receiving the article

- Thorough Double blinded peer review

- Rapid Publication

- Issue of Publication Certificate

- High visibility of your Published work

Website: www.actascientific.com/

Submit Article: www.actascientific.com/submission.php Email us: editor@actascientific.com

Contact us: +919182824667 